\title{
La fille de Louis XVI et l'opinion en 1795 : sensibilité et politique
}

Hélène Becquet

\section{(2) OpenEdition \\ 12 Journals}

Édition électronique

URL : https://journals.openedition.org/ahrf/1620

DOI : 10.4000/ahrf.1620

ISSN : 1952-403X

\section{Éditeur :}

Armand Colin, Société des études robespierristes

\section{Édition imprimée}

Date de publication : 1 septembre 2005

Pagination : 69-83

ISSN : 0003-4436

\section{Référence électronique}

Hélène Becquet, «La fille de Louis XVI et l'opinion en 1795 : sensibilité et politique », Annales historiques de la Révolution française [En ligne], 341 | juillet-septembre 2005, mis en ligne le 15 septembre 2008, consulté le 23 avril 2022. URL : http://journals.openedition.org/ahrf/1620 ; DOI : https://doi.org/10.4000/ahrf.1620

Ce document a été généré automatiquement le 23 avril 2022.

Tous droits réservés 


\title{
La fille de Louis XVI et l'opinion en 1795 : sensibilité et politique
}

\author{
Hélène Becquet
}

Le 10 août 1792, Marie-Thérèse-Charlotte de France (1778-1851), fille aînée de Louis XVI et Marie-Antoinette d'Autriche, suivait ses parents au Temple, dernière demeure de la famille royale. Après avoir vu disparaître tous les membres de sa famille, elle reste seule dans sa prison, séparée de son frère Louis-Charles qui se trouve un étage audessous d'elle. Le silence le plus rigoureux entoure les prisonniers du Temple, jusqu'à la mort du jeune Louis XVII le 8 juin 1795. L'opinion s'émeut alors du sort de Madame Royale. Articles de presse, mémoires, chansons et poèmes se multiplient, réclamant sa libération et chantant ses malheurs. Ces publications sont d'abord des manifestations de la sensibilité de l'opinion publique, de la sensibilité (voire de la sensiblerie) d'une époque qui rejette la Terreur et se réfugie dans le rêve. Madame Royale est alors un personnage romantique, seulement à demi réel. Mais ces écrits sont aussi plus proprement politiques: ils critiquent vigoureusement la Convention et mènent une véritable propagande pour un retour à la monarchie sous couvert de jolies histoires à la mode.

1. Le réveil de l'opinion en faveur de la fille de Louis XVI

2 Selon Mme de Tourzel, son ancienne gouvernante, le décès de Louis XVII « avait fait une grande sensation, et avait opéré un changement sensible dans l'opinion publique qui en accusait les conventionnels ${ }^{1}{ }^{\prime}$. Si l'on peut douter du fait que cette mort ait réellement bouleversé l'opinion publique, il est en revanche certain que le décès du seul héritier légitime de Louis XVI semblait mettre fin à tout espoir de rétablissement de la royauté. Désormais, le sort de Marie-Thérèse de France, inapte à régner selon la loi salique, apparaissait comme tout à fait indépendant du cours de la politique française. Elle devenait, en théorie, un personnage négligeable. Par ailleurs, la mort de son frère, le 20 prairial an III, survient en pleine répression des émeutes de Prairial, à un moment où les derniers Jacobins et sans-culottes sont pourchassés et la presse de gauche muselée. Ces deux facteurs, apparente insignifiance de la princesse et silence de 
la presse de gauche, expliquent l'ampleur du mouvement d'opinion en faveur de MarieThérèse de France.

3 Peu après la mort de Louis XVII, on commence à s'inquiéter de la santé de sa sœur. On la pense à l'agonie, peut-être empoisonnée, comme son frère, par la Convention ${ }^{2}$. Les journaux multiplient les articles sur le sort de Marie-Thérèse, réclamant sa libération au nom de la pitié et de l'humanité. Ainsi les Annales patriotiques, le 26 prairial an III (14 juin 1795):

«La santé de sa sœur est, dit-on, très mauvaise et il est à craindre qu'elle ne suive bientôt son frère au tombeau si l'on ne se hâte de lui faire respirer un air plus salubre.

Tout porte à croire que la Convention nationale, dirigée par des principes d'humanité et de justice, rendra la liberté à cette jeune infortunée dont le seul crime est d'être née d'une race proscrite et qui ne peut devenir dangereuse sous aucun rapport. »

Des brochures, souvent adressées à la Convention, paraissent également pour réclamer sa libération. La première est une pétition de la ville d'Orléans que nous avons trouvée sous le titre, Le premier cri pour la délivrance de Madame Royale, fille de Louis XVI, titre sans doute donné a posteriori. Cette pétition fut publiée dans le journal royaliste modéré les Nouvelles politiques nationales et étrangères du 30 prairial an III (18 juin 1795) qui précise qu'elle aurait été présentée à la Convention. Selon Mallet du Pan, la Convention aurait eu « la bassesse de la dissimuler ${ }^{3}$ ». Pourtant, elle a circulé, au grand mécontentement de certains Orléanais qui protestèrent dans la Sentinelle, journal de Louvet ${ }^{4}$. La ville de Dreux a suivi cet exemple et aurait été refusée à la barre de la Convention ${ }^{5}$. Les sections de Paris auraient également eu le projet de demander à cette assemblée la délivrance de la princesse ${ }^{6}$.

Une Opinion d'un Français sur la détention de Marie-Thérèse-Charlotte de Bourbon, fille de Louis XVI, dernier roi des Français et un Mémoire pour Marie-Thérèse-Charlotte de Bourbon, tous deux parus anonymement, sont destinés, eux aussi, si l'on en croit la page de titre, à la Convention. On peut douter, là encore, que ces ouvrages y aient jamais été présentés mais ils connurent un certain succès. Ainsi, quand Mallet du Pan, alors en Suisse, donne des indications sur la famille royale à l'Empereur, il puise sa source dans ces documents ${ }^{7}$. Le Mémoire est annoncé par voie de presse, dès le 3 messidor (21 juin), dans le Courrier républicain. Il est déposé à la Bibliothèque nationale par crainte des contrefaçons, ce qui n'empêche pas qu'il soit copié quelques jours plus tard ${ }^{8}$.

6 Les arguments sur lesquels ces textes se fondent pour demander la libération de la princesse sont toujours les mêmes. Elle est avant tout présentée comme un être politiquement inoffensif : c'est l'argument clef dont découlent tous les autres. Étant née fille de France, elle ne peut en aucun cas prétendre à la couronne dont la loi salique l'exclut. Les journalistes le rappellent à satiété : les républicains trouvent «dans la monarchie elle-même une garantie contre la monarchie ${ }^{9}$ ». Dans ce cas, la garder en prison n'a plus de sens, c'est même une injustice puisque cela signifie qu'on lui tient rigueur de sa naissance et des crimes de ses parents : un comble, précise-t-on, pour une république dont la principale valeur est l'égalité ! Si Madame Royale ne peut plus être traitée comme une princesse, il faut du moins qu'elle soit traitée comme les autres femmes. On en appelle alors aux «âmes sensibles». On glose sur sa jeunesse et l'abandon dans lequel elle se trouve - nous allons y revenir. On met enfin en avant la fierté nationale : la Nation française, naturellement généreuse, se doit de la libérer. La République fera ainsi preuve d'une magnanimité dont l'histoire se souviendra ${ }^{10}$. 


\section{La Jeune Infortunée}

7 Brochures et articles ne se contentent pas de réclamer la libération de Madame, ils racontent aussi les malheurs de la descendante des deux plus grandes dynasties d'Europe. L'histoire tragique de la princesse est souvent rapportée sous forme de complaintes et de romances chantées sur des airs connus du public. Le directeur de la Quotidienne, Joseph-François Michaud, en a fait un recueil, sous la forme d'un almanach intitulé Les adieux de Marie-Thérèse-Charlotte de Bourbon, publié pour la première fois à Bâle en 1796 et republié en 1797, sous le nom de M. d'Albins. Sa diffusion semble avoir été importante, le Courrier Républicain du 26 janvier 1797 précise qu'on se «l'arrache » ${ }^{11}$. Ces textes brossent le portrait d'une jeune fille malheureuse et souvent triste, que l'on nomme désormais « la Jeune Infortunée ».

8 La princesse est, comme il se doit, jeune et belle, ce qui rend son sort plus touchant encore. Pour Claude-François Beaulieu, elle est « belle, dit-on, comme la rose qui vient d'éclore ${ }^{12}$ ", et, ajoute l'auteur de l'Opinion d'un Français sur Marie-Thérèse-Charlotte de Bourbon, " elle fut belle autrefois de l'éclat des grandeurs, elle est plus belle aujourd'hui de son infortune, semblable à ces étoiles qui jettent une plus vive lumière en tombant de la voûte des cieux ${ }^{13}$ ". Ces louanges peuvent prendre des formes allégoriques: Marie-Thérèse devient la rose du Temple ou une blanche colombe persécutée. Ces thèmes appartiennent au registre allégorique utilisé traditionnellement pour les filles de France et simplement adapté aux circonstances : dès sa naissance, on avait fait d'elle une rose, ornement de la dynastie et une colombe, symbole de pureté et de douceur.

9 Tout ce que les journaux publient de la vie quotidienne de Marie-Thérèse vient renforcer cette image d'une princesse «idéale", modeste, patiente et douce. La manière dont sa vie est décrite contribue à faire d'elle l'héroïne d'un conte de fées ou de quelque récit légendaire. Le public connaît la vie de la princesse à travers ce que Michaud appelle, dans son almanach sur Marie-Thérèse, « les bulletins du Temple ». On ne sait pas exactement d'où proviennent ces «bulletins », publiés dans les journaux de droite au rythme d'environ un par mois. Peut-être la famille de Mme de Chanterenne, compagne de Madame Royale au Temple, a-t-elle fourni des indications ${ }^{14}$. On ne sait pas non plus qui écrit ces articles. Celui relatant la visite de Mme de Tourzel est signé Alexandre Raimond mais rien ne prouve que cet écrivain, dont nous ne savons rien par ailleurs, ait été l'auteur des autres. Michaud, dans Les adieux de Marie-Thérèse-Charlotte de Bourbon, les publie manifestement tous : le premier date du 10 août 1795, le dernier du 19 décembre 1795. Entre ces deux dates, six bulletins s'échelonnent que l'on retrouve dans les journaux selon des découpages divers.

Différentes scènes sont dépeintes dans ces courts comptes rendus de la vie de MarieThérèse. On se préoccupe notamment des personnes qui lui rendent visite, ainsi l'ancienne gouvernante des Enfants de France, Mme de Tourzel et sa fille Pauline :

« La fille de Louis XVI était au jardin lorsque madame de Tourzel et madame sa fille $\mathrm{y}$ arrivèrent.

Avec quel empressement la prisonnière courut à elles, se précipita dans leurs bras, pressa la jeune dame contre son cœur ! Elle avait été la première compagne, la plus tendre amie de son enfance.

Quel tableau touchant! Quel attendrissant spectacle! Aucun de ceux qui en furent témoins ne put retenir ses larmes: elles étaient délicieuses: c'était celles du sentiment! Que n'avez-vous pu, à la distance où vous étiez, les voir couler, ô vous, fille de tant de rois! Vous! Que je ne saurais appeler d'aucun nom qui convienne à vos malheurs, à tout le respect qui leur est dû, et à la tendresse avec laquelle vous chérit toute âme susceptible d'humanité ! ... Mais, du moins, vous ne pouvez ignorer 
tout l'intérêt qu'on prend à vous voir... Oh! Non, vous ne pouvez l'ignorer...

combien votre personne est chère ${ }^{15}$ !»

11 Ces tableaux sentimentaux devaient effectivement susciter bien des larmes puisque, quelques semaines plus tard, un autre bulletin du Temple relatait les retrouvailles de Madame Royale et de Mme de Mackau, sa sous-gouvernante. Ces récits exaltent la bonté de la princesse, son affection pour les personnes qui ont entouré son enfance. Dans ces bulletins, on cherche avant tout à émouvoir le lecteur. Ainsi, on sait peu de chose, finalement, sur la visite de Mme de Tourzel et de sa fille. Le seul élément qui intéresse l'auteur est le fait que Madame serre Pauline dans ses bras. L'action se concentre en deux lignes. Le reste de l'article souligne simplement l'effet pathétique de cette rencontre et l'explicite au lecteur. Il faut conquérir le public: le cœur de toute personne «susceptible d'humanité» doit s'attendrir à la vue du bonheur de la princesse.

àtosité à l'égard de Madame Royale ne s'arrête pas aux visites, elle s'étend à tous les détails de son quotidien. On publie la liste des livres qui lui sont fournis $\left({ }^{16}\right)$, on précise son habillement aux différents moments de la journée. Voici le portrait que l'on fait d'elle :

«Madame Marie-Thérèse-Charlotte de Bourbon paraît jouir d'une assez bonne santé ; elle est d'une taille avantageuse ; ses traits qui étaient, il y a trois ans, forts délicats, ont pris un beau caractère ; ses yeux sont grands, son teint paraît un peu bruni ; ses cheveux, de blonds qu'ils étaient, sont devenus un peu châtains ; elle les porte habituellement sans poudre et noués par derrière; sa coiffure est ordinairement un fichu attaché par un nœud sur le devant qui forme la rosette ${ }^{17}$. »

13 Cette chevelure semble avoir déchaîné les passions puisque le bulletin suivant, du 15 octobre, précise aux «élégantes» que Madame ne porte pas de perruque, contrairement à ce que certaines prétendaient: sa belle chevelure est totalement naturelle ${ }^{18}$.

Deux personnages pittoresques apparaissent dans cette histoire et contribuent à faire de la princesse un personnage hautement romanesque ${ }_{20}^{[0}$ : une chèvre et surtout le chien Coco que les gardiens du Temple avaient donné à Louis XVII pour le distraire. Très vite, ces animaux deviennent des emblèmes de la fidélité, sans cesse opposés à l'ingratitude et à la méchanceté des hommes.

La vie quotidienne de la princesse est ainsi évoquée sous des couleurs plutôt gaies. Madame Royale règne sur un petit royaume imaginaire dont la tour du Temple est le palais. Le passé y est évoqué seulement par allusions et sans que l'on s'y attarde sinon pour insister sur le fait que l'amélioration de la vie de la princesse est un juste retour des choses.

Cependant, le sort de Madame Royale est fréquemment montré sous un jour plus sombre. Dans les poèmes et les chansons dont la princesse est le sujet, la Jeune Infortunée pleure bien souvent sur son sort. La tour du Temple n'est plus le cadre des rires et des jeux de la jeune fille mais redevient une sinistre prison. Son aspect médiéval, massif et froid la rend propre aux descriptions mélancoliques. Elle en devient presque un personnage à elle seule.

On montre la princesse se consumant de tristesse dans cette sinistre tour. Elle ne cesse de songer au décès de ses proches et redoute sa propre mort tout en la considérant comme la seule issue possible à sa situation. Ce désespoir de Marie-Thérèse est un leitmotiv des écrits sur la princesse. Une romance de cette veine un peu lugubre, 
Pétition d'une jeune infortunée, a connu un succès certain. Elle a été publiée dans maints journaux, dont le très modéré Journal de Paris. Elle a été mise en musique sur deux airs différents, autre preuve de l'intérêt qu'elle a provoqué. Dans cette œuvre et dans d'autres du même genre, Marie-Thérèse est une véritable héroïne romantique. Elle en a le destin tragique. Persécutée depuis des années, privée de sa famille, elle attend la mort. Elle en a le physique : blonde et affaiblie par la prison, on l'imagine diaphane, mélancolique ${ }^{19}$. Un tableau qui la représente sans doute à cette période, attribué à Greuze, correspond à cette image ${ }^{20}$. Elle n'est pas parfaitement belle, la tristesse se lit sur son visage, le voile de deuil flotte sur ses épaules rappelant qu'elle est «seule au milieu des ténèbres, des tombeaux et des ombres ${ }^{21}$ ».

Une autre image, liée à celle-ci, est en effet récurrente: celle du tombeau. La " poétique des tombeaux ", venue des auteurs anglais du début du siècle, connaît un vif succès en France après la Terreur. Les morts violentes, les violations de sépultures, royales en particulier, les inhumations en fosses communes ont contribué à cet engouement ${ }^{22}$. Dans le cas de Madame Royale, la princesse devient symboliquement la seule gardienne $\mathrm{du}$ tombeau de ses parents.

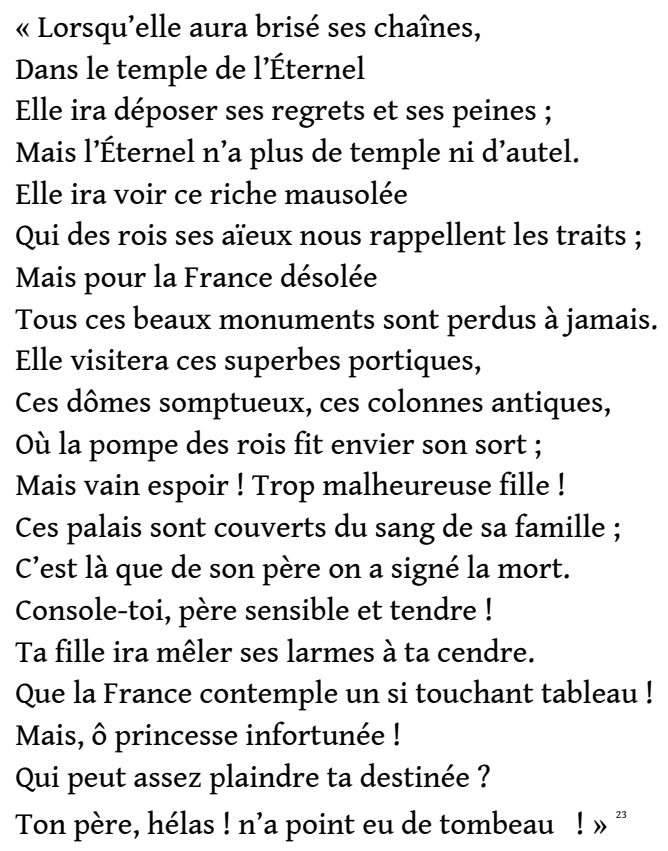

Cette jeune fille qui se doit à la mémoire de ses parents prend alors les traits de Selma, personnage issu des chants d'Ossian, chantant la mort de son père Fingal, la lune comme seul témoin. C'est la première assimilation de Madame à un personnage littéraire. Elle prend ainsi une dimension nouvelle plus lointaine et plus familière à la fois. Sous ces couleurs appréciées à l'époque, ces poèmes mettent en valeur la piété filiale. La « Jeune Infortunée » chante son désespoir mais elle chante surtout la mort de son père, première à rendre un culte à ses cendres.

Par le biais de ces poèmes et de ces chansons, la fille de Louis XVI, ainsi qu'on l'appelle généralement, devient une héroïne romantique. La princesse n'est plus tant Madame Royale que la "Jeune Infortunée ». Indépendamment de ce qu'est réellement MarieThérèse, naît une image d'elle-même qu'elle ne contrôle pas. Cette image se répand largement dans le public et devient plus particulièrement l'objet de l'admiration respectueuse des royalistes.

3. La rotonde du Temple 
21 Certaines manifestations sont en effet plus clairement propres aux royalistes, tout en restant dans le même ordre d'imagerie sentimentale. Les fidèles de la monarchie défunte alimentent sans cesse le courant d'opinion favorable à la princesse dont ils forment le cœur.

Le 12 thermidor an III (30 juillet 1795), Mme de Chanterenne demande au Comité de Sûreté générale l'autorisation pour Madame de se promener, pour raisons de santé. La permission est bientôt accordée.

«À peine Mme Cléry, dont le mari était auprès de Sa Majesté Louis XVIII, eut-elle appris que Madame se promenait le soir, qu'elle s'empressa de louer dans une maison, appelée la rotonde du Temple, deux chambres dont les croisées donnaient sur le jardin, la rue qui sépare étant fort étroite. De là, on découvrait, on entendait parfaitement. On porta une harpe dans la pièce que nous nommions la salle $d u$ concert; et la première fois on chanta des morceaux qui n'avait point un rapport direct avec la situation de Madame. Pour le concert suivant, je composai la romance intitulée Les Regrets, dont Madame Cléry fit la musique et que son amie chanta 24. »

Les versions, à ce sujet, divergent, Hue prétendant lui aussi être à l'origine de ces réunions ${ }^{25}$. Toujours est-il que ces réunions avaient bien lieu et que l'on y chantait son dévouement à la princesse. Dans les romances chantées dans la rotonde que nous avons pu lire, ce n'est pas la princesse qui parle mais ses serviteurs fidèles dont Lepître et Hue sont des prototypes. Rappelons que le premier, officier municipal désigné pour garder la famille royale au Temple, a tenté de faire évader la reine, et que le second, valet de chambre de Louis XVI, a d'abord été emprisonné avec son maître puis a été arrêté en raison de ses liens avec le roi. Les «concertistes » du Temple disent à Madame leur indéfectible fidélité, en se comparant, entre autres, au troubadour Blondel qui venait chanter au pied de la tour où Richard Cœur de Lion était prisonnier pour le consoler et lui porter des nouvelles ${ }^{26}$. Les chansons servaient également à informer Marie-Thérèse de ce qui se passait à l'extérieur: la Consolation de Lepître lui apprend ainsi que l'Autriche a accepté les conditions pour sa libération ${ }^{27}$.

Ces concerts connaissent un succès croissant. Aux anciens serviteurs de la famille royale, Lepître, Hue, Mme Cléry, Mme de Tourzel, viennent s'ajouter bien d'autres personnes. Les places sont chères parce qu'elles sont trop peu nombreuses pour accueillir tout le monde. Aussi un deuxième appartement est-il loué, rue de Beaujolais, au grand agacement de la police qui, quoi qu'en dise Hue, n'aimait pas beaucoup ces manifestations ${ }^{28}$. Un rapport signé par un certain Leblanc indique la présence d'une centaine de personnes qui changent souvent mais qu'il qualifie, sans aucune hésitation, de royalistes ${ }^{29}$. Le portrait qu'il en dresse contraste un peu avec celui d'Allonville qui prétend que s'y trouvent des gens de tous états. On peut supposer qu'à des fidèles, des gens de «l'ancienne cour » comme le dit Leblanc, se joignent plus occasionnellement divers curieux. En tout cas, il ne semble pas que ce mouvement, d'une certaine importance, ait été monopolisé par un parti quelconque. Constitutionnels et absolutistes devaient se croiser dans cette rotonde, nobles et petits bourgeois également ${ }^{30}$. Ce sont l'émotion et la sensibilité qui regroupaient tous ces gens.

On chante donc mais on peint aussi. Le comte de Paroy a fait passer à la princesse un dessin de la tour du Temple ainsi qu'une boîte de crayons ${ }^{31}$. Quant au citoyen Macret, il l'a dessinée après l'avoir observée au télescope, puis en a tiré une gravure dont la publication est annoncée dans le Courrier républicain ${ }^{32}$. Les nombreux exemplaires trouvés dans les collections de la B.N.F. laissent supposer qu'elle a été beaucoup 
diffusée. La lettre donnait cette indication précieuse qui faisait toute la valeur de l'estampe : «dessinée d'après nature ». Ce portrait, revendiqué comme le plus fidèle possible, était ainsi une manière d'appréhender la personnalité de Marie-Thérèse.

Le gouvernement s'inquiète de ces réunions, au vu des proportions qu'elles ont prises :

«Cependant la police prit de l'humeur; elle nous intima l'ordre de cesser les concerts. Ce fut surtout le jour de la Saint-Louis, fête à laquelle des couplets faisaient allusion, qu'éclatèrent des plaintes : elles devinrent si vives, les menaces si pressantes, qu'il fallut obéir. Madame partit peu de temps après ${ }^{33}$. »

Lepître est peu précis sur les dates, Madame de Tourzel indique que "après le 13 vendémiaire, il ne fut plus possible de [s']exprimer aussi visiblement ${ }^{34} »$. On peut en déduire que ces concerts auront duré environ deux mois, ceux d'août et septembre $1795{ }^{35}$. Leur arrêt après l'insurrection du 13 Vendémiaire n'a rien de bien surprenant, la répression qui s'abattit sur les royalistes les ayant poussés à davantage de prudence.

4. Significations politiques

Le destin de Madame Royale, raconté avec force détails pathétiques, doit susciter la pitié de l'opinion publique. Alexandre Raimond dans sa Lettre à Mme Chanterel prétend ainsi « qu'il n'est pas en France un individu (les assassins exceptés) qui ne la plaigne sincèrement ». On comprend aisément que la parenthèse s'adresse à un type bien particulier d'assassins, ceux qui ont porté la main sur la famille royale et qui continuent à en persécuter la seule survivante. Autrement dit, ce terme désigne, en partie au moins, les conventionnels - qu'ils aient été vraiment responsables ou non n'a ici pas d'importance. La plupart des textes dont nous avons parlé désignent implicitement la Convention thermidorienne comme responsable des malheurs de la princesse, puisque, même si elle n'a pas ordonné son emprisonnement, elle le cautionne en refusant la libération de Madame.

Mais, au-delà de la Convention, c'est la Révolution elle-même qui est mise en accusation. C'est en effet la Révolution qui a arraché Marie-Thérèse à son destin doré de fille de France, l'a rendue orpheline et l'a enfermée. En outre, dans nombre de ces ouvrages, les révolutionnaires sont présentés comme des êtres abjects qui maltraitent des enfants innocents. Les notes du Mémoire de Beaulieu sont à cet égard exemplaires. Que peut-on attendre d'une Révolution responsable d'un grand nombre de morts et du malheur d'une jeune fille? Telle est la question que semblent poser les ouvrages et articles concernant Marie-Thérèse-Charlotte de France. Plaindre Madame Royale c'est considérer que, sous un certain nombre de ses aspects, la Révolution est critiquable, voire condamnable.

30 Une partie de cette production est donc clairement de la propagande contrerévolutionnaire. Certes, certains textes ont pu être écrits spontanément. Les romances, par exemple, obéissent aussi à une sorte d'effet de mode. Cependant, l'impulsion est bien venue des journaux et, là, la spontanéité n'a plus vraiment sa place. Savoir qui étaient ces journalistes, qui étaient les auteurs de ces brochures permet de donner un éclairage intéressant au mouvement d'opinion créé autour de Madame.

31 Il faut tout d'abord souligner une chose, c'est la grande influence de la presse de droite qui domine réellement l'opinion publique à cette période, en raison notamment de la répression anti-jacobine qui suivit les journées de Prairial ${ }^{36}$. La multiplicité des titres est compensée par une sorte de ligne éditoriale commune, définie entre 1794 et 1797 lors de réunions régulières. Dès Thermidor, les journalistes se réunissent chez un restaurateur place du Louvre. Sous l'égide des frères Bertin, propriétaires du Journal des 
Débats, se retrouvent, entre autres, les constitutionnels Dussault, rédacteur de L'Orateur du Peuple, Lagarde du Journal de Perlet, Charles Lacretelle, les royalistes purs RicherSérizy, auteur de l'Accusateur public, Joseph Michaud, rédacteur de la Quotidienne, Hyde de Neuville et Joseph Fiévée. En sont exclus les modérés comme Roederer, propriétaire du Journal de Paris ${ }^{37}$. Lorsque l'on constate la simultanéité des articles demandant la libération de Madame Royale, on ne peut que penser que leur rédaction a été décidée au cours d'une de ces réunions. Le tirage de ces journaux est élevé : 8000 par jour pour la Quotidienne en 1796. Ils se diffusent en outre un peu partout en province, chaque région ayant ses titres de prédilection ${ }^{38}$. Ils ont donc un impact certain. Ils avaient les moyens de faire naître un vrai courant « populaire » autour de Madame Royale ${ }^{39}$.

Mais le plus intéressant est le fait que ces journaux royalistes ont entraîné dans leur sillage des journaux qui, en principe, ne le sont pas. Les Annales patriotiques et littéraires de Louis-Sébastien Mercier en sont un exemple. Mercier a été girondin et a fait partie des soixante-quinze députés exclus de la Convention après l'arrestation des meneurs de sa faction. C'est donc, a priori, un républicain, contrairement aux journalistes que nous avons cités, mais il n'a pas voté la mort du roi ${ }^{40}$. Il fut emprisonné et libéré après le 9 Thermidor ce qui lui permit de reprendre la rédaction de son journal, dans un sens plutôt réactionnaire. Il employait par exemple Ange Pitou, chansonnier royaliste, qui devint un peu plus tard agent de Louis XVIII, pour rédiger les comptes rendus de la Convention. Leur collaboration remontait en fait à une affaire un peu compliquée qui consistait à faire entrer Ange Pitou dans le comité de rédaction de l'Ami du peuple, ancien journal de Marat, afin de donner à ce journal un tour suffisamment extrémiste pour discréditer définitivement les Jacobins ${ }^{41}$. Ces rapports entre les deux hommes montrent que la lutte commune contre les "terroristes " rapprochait royalistes et modérés ${ }^{42}$. On observera d'ailleurs que les articles du journal de Mercier sur la princesse paraissent au même moment que ceux des journaux royalistes. Un autre journal suit également le mouvement, le Journal de Paris qui publie notamment la Pétition d'une jeune infortunée dès sa parution. La personne de la princesse est suffisamment émouvante, sa cause paraît suffisamment juste pour gagner de proche en proche un grand nombre de partisans.

On peut observer le même phénomène en examinant le parcours des auteurs des brochures sur la princesse, auteurs souvent issus des mêmes milieux de journalistes. Le cas de Joseph-François Michaud est remarquable. C'est lui qui a regroupé les écrits sur Marie-Thérèse dans l'almanach, Les adieux de Marie-Thérèse-Charlotte de Bourbon. Il a sans doute écrit une partie des pièces qui y sont insérées mais il est impossible de savoir lesquelles. Michaud a fait partie des défenseurs du roi le Dix Août aux Tuileries et a dû se cacher par la suite. Après le 9 Thermidor, il travaille au Courrier républicain, journal royaliste, contrairement à ce que laisserait supposer son nom. Puis il fonde avec Rippert de Beauregard et Leriche, le Tableau de Paris qui deviendra la Quotidienne, journal royaliste des plus intransigeants ${ }^{43}$. Ses positions politiques le feront emprisonner plusieurs fois et lui vaudront d'être condamné à mort après l'insurrection de Vendémiaire. Son respect pour Marie-Thérèse et son ardeur à la défendre se comprennent donc aisément. Claude-François Beaulieu, auteur du Mémoire pour MarieThérèse-Charlotte de Bourbon a connu les mêmes vicissitudes que Michaud avec lequel il travaillait à la Quotidienne: défense du roi au Dix Août, emprisonnement sous la Terreur, proscription à la suite de Vendémiaire et, plus tard, après Fructidor. Sous la Restauration, le premier obtiendra une charge de lecteur du roi, le second sera 
pensionné. Ils forment le «noyau dur » des défenseurs de la princesse et sont partisans d'une monarchie absolue. Ils sont les auteurs des textes les plus politiques sur Madame.

L'auteur de la pétition d'Orléans, Gabriel-Jacques Laisné de Villevêque appartient, lui, à la tendance constitutionnelle. Son profil est atypique car, à l'époque où il envoie cette pétition à la Convention, il n'est ni homme de lettres ni homme politique. Ce n'est qu'en 1800 qu'il fait partie du Conseil général du Loiret où il reste en place durant tout l'Empire. C'est un négociant dont les opinions sont d'un royalisme très modéré, comme il le montrera dans sa carrière de député sous la Restauration qu'il n'interrompra d'ailleurs pas en 1830. Sa démarche pour la délivrance de Madame Royale surprend donc un peu. Sans doute faut-il y voir l'expression la plus proche de l'état de l'opinion modérée en 1795 , s'attendrissant sincèrement, non sans nostalgie, sur les malheurs de la fille de Louis XVI, sans faire d'elle un étendard politique. Jacques Lablée, autre littérateur qui s'est intéressé à la princesse, partage sans doute la même sensibilité et les mêmes opinions. Fils d'un négociant en vin, il fut garde national, administrateur de la Commune de Paris et président de la section du Luxembourg. Un révolutionnaire engagé donc, mais emprisonné pour avoir rédigé un journal anti-jacobin ${ }^{44}$. Libéré avec Thermidor, il se consacre entièrement aux lettres et compose une romance sur Madame qu'il publie dans ses mémoires, La Jeune Captive ${ }^{45}$. De nouveau, dans cette œuvre, c'est l'aspect romantique de la princesse qui l'emporte, sans que l'on sache réellement quelles pensées politiques animaient son auteur.

Le courant en faveur de Madame Royale n'est donc pas anodin. Les personnes qui écrivent sur les malheurs de la princesse sont en grande majorité de sensibilité royaliste. D'ailleurs, le mouvement marque le pas après l'insurrection de Vendémiaire parce que beaucoup d'entre elles sont emprisonnées ou obligées de se cacher. L'aura romantique de la princesse a considérablement servi le parti royaliste. Un consensus pouvait s'établir entre les modérés et les royalistes de tous bords autour de cette figure émouvante. Des écrivains qui n'étaient pas nécessairement royalistes se sont laissés porter par le mouvement, mais ils n'en servaient pas moins, consciemment ou non, la cause monarchique. Le but de ces diverses publications était certes la libération de Marie-Thérèse mais, au-delà, en faisant l'apologie de la princesse, c'était la Convention responsable de ses malheurs que l'on attaquait. La campagne de dénigrement de la Convention qui a précédé le 13 Vendémiaire est ainsi passée par la figure de MarieThérèse de France. Plus largement, réclamer la libération de Madame, chanter ses souffrances, c'était favoriser une restauration.

5. La libération

Tous les écrits étudiés réclamaient la libération de Marie-Thérèse. Pourtant, celle-ci n'eut lieu que de longs mois après le début de cette campagne de presse. Dans la séance du 12 messidor an III (1er juillet 1795), Treilhard, au nom des Comités de Salut public et de Sûreté générale, propose un décret imposant à l'Autriche la libération des députés et ministres français qu'elle détient en échange de la princesse ${ }^{46}$. Marie-Thérèse serait ainsi échangée contre Beurnonville, ministre de la Guerre et son domestique, Marchand, les députés Bancal, Lamarque, Camus, Quinette, Drouet, les secrétaires nationaux Faucon et Villemar, Manoire, adjudant de Beurnonville, les ambassadeurs Constant-Benuveau, Semonville et Maret. Ce décret est aussitôt voté par la Convention. La décision est unilatérale, aussi la réaction autrichienne est-elle assez embarrassée dans un premier temps, d'autant qu'elle n'a guère envie de s'encombrer de la princesse même si elle est une petite-fille de l'impératrice Marie-Thérèse. 
Il fallut attendre plus d'un mois avant que l'Empereur ne se décide à répondre, de manière positive, par le biais du général Clairfayt. Le Comité de Salut public désigne alors, le 27 thermidor an III, le premier secrétaire de l'ambassade de Suisse, Bacher, pour les négociations qui auront lieu à Bâle ${ }^{47}$. Celles-ci furent particulièrement longues, en raison des exigences autrichiennes et de la situation politique perturbée de la France. Pendant ce temps, à Paris, on s'entretenait beaucoup de cette libération que l'on croyait toujours imminente ${ }^{48}$. Les journaux royalistes et modérés ne manquaient pas de mentionner les préparatifs faits en Autriche dans l'attente de l'arrivée de la princesse ${ }^{49}$. Ce n'est que dans la nuit du 18 au 19 décembre 1795 que Marie-Thérèse de France est conduite à Bâle pour y être remise à son cousin François II. Un long exil commençait.

\section{NOTES}

1.Louise-Elisabeth de CROY D'HAVRE, duchesse de TOURZEL, Mémoires, Paris, Mercure de France, 1969, p. 430.

2.Adolphe SCHMIDT, Tableaux de la Révolution française, Leipzig, 1870, 4 vols, vol 2, rapport de police du 29 prairial an III (16 juin 1795). Alphonse AULARD, Paris pendant la Réaction thermidorienne et sous le Directoire, Paris, 1898, t. II, rapport de police du 29 prairial an III, p. 18.

3.Jacques MALLET DU PAN, Correspondance inédite avec la cour de Vienne, Paris, 1884, éd Michel ANDRÉ, 2 vols, t. II, lettre du 23 juin 1795, pp. 242-243. Nous n'avons par ailleurs trouvé trace de cette pétition ni dans la série $\mathrm{C}$ des Archives nationales ni dans le Moniteur.

4.La Sentinelle, 15 messidor an III (3 juillet 1795).

5.Le procès des Bourbons, Paris, 1798, 2 vols, t. II, pp. 424-425. Sous la Restauration, la ville est félicitée pour ce geste.

6.Jacques MALLET DU PAN, op. cit., lettre du 23 juin 1795, pp. 242-243. Mais BarruelBeauvert, qui fit une démarche similaire de la part de Mantes peu avant Vendémiaire, dit s'être heurté à la résistance des sections. Le personnage devait inspirer bien plus de méfiance que le projet en lui-même, voir comte Antoine-Joseph de BARRUEL-

BEAUVERT, Lettres sur quelques particularités secrètes de l'histoire pendant l'interrègne des Bourbons à M. le comte A. ***, Paris, 1815, 3 vol. , vol. 1, pp. 299-307.

7.Jacques MALLET DU PAN, op. cit., t. II, lettre du 23 juin 1795.

8.Courrier républicain, 4 et 9 messidor an III ( 22 et 27 juin 1795).

9.Opinion d'un Français sur la détention de Marie-Thérèse-Charlotte Bourbon, fille de Louis XVI, ci-devant roi des Français, dans Mémoire adressé à la Nation pour Marie-Thérèse-Charlotte de Bourbon, suivi d'une opinion adressée à la Convention nationale pour la fille de Louis XVI, pour Louise-Marie-Adélaïde Bourbon d'Orléans et Louise-Thérèse Bathilde Bourbon d'Orléans ; accompagné de notes curieuses et intéressantes sur la prison de Marie-Antoinette d'Autriche et sur les autres prisonniers du Temple, Paris, 1795, p. 12. 
10.On retrouve cette réflexion dans les conversations rapportées par les agents de police, sans doute en écho aux articles des journaux ; voir Alphonse AULARD, op. cit., t. II, rapport du 5 messidor an III (23 juin 1795), p. 30.

11.Linda A. LAPOINTE, «L'image contre-révolutionnaire : l'hagiographie royale », Études françaises, Montréal, 1989, p. 230.

12.Claude-Francois BEAULIEU, Mémoire pour Marie-Thérèse-Charlotte de Bourbon, fille de Louis XVI, ci-devant roi des Français, retenue à la tour du Temple, dans Mémoire adressé à la Nation..., op. cit., p. 7.

13.0pinion d'un Français..., op. cit., p. 10.

14.Dans un bulletin, daté du 30 septembre dans l'almanach de Michaud, les renseignements donnés sur Mme de Chanterenne proviennent de sa famille. Peut-être en était-il ainsi pour d'autres renseignements concernant la princesse. Mme de Chanterenne avait été choisie par la Convention, à la fin du mois de juin 1795, pour tenir compagnie à Madame Royale.

15. Courrier républicain, 21 fructidor an III (7 septembre 1795).

16.Id., 11 fructidor an III (28 août 1795).

17.M. D’ALBINS, Les adieux de Marie-Thérèse de Bourbon, Bâle, 1797, bulletin du 30 septembre 1795, pp. 124-125.

18.Ibid., pp. 126-127.

19.Sur l'héroïne romantique, voir André MONGLOND, Le Préromantisme français, réimp. Genève, Slatkine, 2000, 2 vol., t. I, pp. 221-223.

20.Nous ne sommes pas d'accord avec la datation proposée par le propriétaire du tableau, Alfred Prost. Il avance 1793 sans argumenter autrement que par le physique de la princesse. Or, il existe un tableau de Louis XVII, daté de 1795 selon François

LAURENTIE, dans son Iconographie de Louis XVII, pp. 24-25. Compte tenu de l'habitude de représenter en même temps les deux enfants, nous pensons que le portrait de MarieThérèse doit être daté de la même année. Voir Jean-Charles-Alfred PROST, Deux œuvres de Greuze, Madame Royale à la prison du Temple, Monseigneur le Dauphin au musée de Besançon, Paris, Firmin-Didot, s.d., 76 p.

21.Claude-Francois BEAULIEU, op. cit., p. 5

22.Paul VAN TIEGHEM, La poésie de la nuit et des tombeaux en Europe au XVIIIe siècle, réimp. Genève, Slatkine, 1970, pp. 161-162.

23.M. D'ALBINS, op. cit., Les chagrins de Marie-Thérèse-Charlotte en sortant du Temple, pp. 60-61.

24.Jacques-François LEPITRE, Quelques souvenirs ou notes fidèles sur mon service au Temple depuis le 8 décembre 1792 jusqu'au 26 mars 1793, Paris, 1814, p. 80.

25.Baron François HUE, Souvenirs, réimp. Oxford, Pergamon Press, 1989, p. 197.

26.Ibid., p. 197 ; Jacques-François LEPITRE, op. cit., p. 92.

27.Ibid., p. 83.

28.Hue prétend que la police était d'accord. C'était peut-être le cas au début quand ces manifestations étaient circonscrites mais leur ampleur a sans doute désagréablement surpris. François HUE, op. cit., p. 199.

29.AN, F7 4392.

30.N'oublions pas que Lepître, à l'origine du mouvement était simple professeur de rhétorique et maître de pension. Par ailleurs, si ces concerts ont duré plusieurs mois, avec un changement fréquent de spectateurs, il est évident que tous les partis y sont représentés, compte tenu de l'importance des constitutionnels dans cette période précédant Vendémiaire. 
31.Jean-Philippe-Gui LE GENTIL, comte de PAROY, Souvenirs d'un défenseur de la famille royale pendant la Révolution (1789-1797), Paris, Plon et Nourrit, 1895, pp. 454-456.

32.Linda A. LAPOINTE, op. cit., p. 231.

33.Jacques-François LEPITRE, op. cit., p. 85.

34.Louise-Elisabeth de CROY d'HAVRE, duchesse de TOURZEL, op. cit., p. 426.

35.Leblanc dans son rapport de police, non daté, indique quatre mois, ce qui paraît surprenant.

36.Henri ZIVY, Le 13 vendémiaire, Paris, F. Alcan, 1900, p. 19.

37.F. GENDRON, La jeunesse dorée, épisodes de la Révolution française, Québec, Presses de l'Université du Québec, 1979, p. 39 ; Jérémy D. POPKIN, The Right Wing Press in France, 1792-1800, Chapel-Hill, 1980, pp. 29-30.

38.Jeremy D. POPKIN, op. cit., pp. 75-82.

39. Compte tenu du fait que les lecteurs de journaux se recrutent essentiellement dans la bourgeoisie : les journaux sont fort chers et ne s'obtiennent que par abonnement. 40.Cf article « Louis-Sébastien Mercier », dans George GRENTE, François MOUREAU, Dictionnaire des lettres françaises : le XVIIIe siècle, Paris, le livre de poche, « la Pochothèque $"$.

41.Fernand ENGERAND, Ange Pitou, agent royaliste et chanteur des rues, Paris, E. Leroux, 1899, pp. 83-87.

42.Mercier aurait accepté l'idée d'une restauration de Louis XVII avec un gouvernement constitutionnel, cf. Fernand ENGERAND, op. cit., pp. 88-89.

43.Jeremy D. POPKIN, op. cit., p. 20.

44.André MONGLOND, op. cit., t. II, pp. 270-275. Monglond le présente comme étant typique de l'écrivain préromantique en France.

45.Jacques LABLEE, Mémoires d'un homme de lettres, Paris, 1824, pp. 221-222.

46.Le Moniteur, 13 et 15 messidor an III (1er et 3 juillet 1795).

47.Le Moniteur, 14 fructidor an III (31 août 1795).

48.Alphonse AULARD, op. cit., t. II, rapports du 21 thermidor an III (8 août 1795), p. 145, du 27 thermidor an III (14 août 1795), p. 162, du 1er fructidor an III (18 août 1795), p.

173.

49.Voir, entre autres, les Annales patriotiques et littéraires, 16 et 18 fructidor an III (2 et 4 septembre 1795), 30 brumaire an IV (21 novembre 1795), 9 frimaire an IV (30 novembre 1795).

\section{RÉSUMÉS}

Marie-Thérèse-Charlotte de France, fille de Louis XVI, emprisonnée au Temple depuis 1792, devient, après la mort de son frère Louis XVII le 8 juin 1795, un objet de compassion. De nombreux articles, des brochures demandent à la Convention nationale sa libération, entraînant un véritable mouvement de l'opinion publique en faveur de la princesse. À travers romances et articles, cette princesse devient une héroïne romantique. Cependant, au-delà de cet aspect sentimental, la louange de Madame Royale cache une condamnation de la Révolution. Ce mouvement en faveur de Marie-Thérèse n'était en effet pas dû au hasard. Le personnage de 
Madame Royale a contribué à unir modérés et royalistes dans la lutte qui les opposait à la Convention nationale et qui déboucha sur le 13 vendémiaire.

Louis XVI's daughter and the public opinion in 1795: sensitivity and politics. Marie-Thérèse Charlotte of France, Louis XVI's daughter, in the Temple prison since 1792, becomes an object of compassion after the death of her brother Louis XVII on the 8th of June 1795. Numerous articles, booklets address the Convention and plead for her release, resulting in a reaction of the public opinion in the favour of the Princess. Through songs and articles she becomes a romantic heroine. Nevertheless, beyond this sentimentality, the praise of Madame Royale hides the condemnation of the Revolution. This reaction in favour of Marie-Thérèse played an important part in uniting moderates and royalists to fight against the National Convention. This will lead to the 13th Vendémiaire uprising.

\section{INDEX}

Mots-clés : Contre-Révolution, opinion publique, journaux, Marie-Thérèse-Charlotte de France (1778-1851), royalisme, Romantisme, journalistes

\section{AUTEUR}

\section{HÉLÈNE BECQUET}

Archiviste-Paléographe, I.H.R.F. - Université Paris I, 17 rue de la Sorbonne, 75005 Paris 